\title{
BMJ Global Health Codevelopment of COVID-19 infection prevention and control guidelines in lower-middle-income countries: the 'SPRINT' principles
}

\author{
Victoria Haldane, ${ }^{1}$ Savithiri Ratnapalan, ${ }^{2,3}$ Niranjala Perera, ${ }^{2,3}$ Zhitong Zhang, ${ }^{2}$ \\ Shiliang Ge, ${ }^{4}$ Mia Choi, ${ }^{5}$ Lincoln L Lau (1) ,2,5 Sudath Samaraweera, ${ }^{6}$ \\ Warren Dodd, ${ }^{7}$ John Walley, ${ }^{8}$ Xiaolin Wei $^{2}$
}

To cite: Haldane V,

Ratnapalan S, Perera N, et al. Codevelopment of COVID-19 infection prevention and control guidelines in lower-middleincome countries: the 'SPRINT' principles. BMJ Global Health 2021;6:e006406. doi:10.1136/ bmjgh-2021-006406

Handling editor Seye Abimbola

- Additional supplemental material is published online only. To view, please visit the journal online (http://dx.doi.org/10. 1136/bmjgh-2021-006406)

Received 26 May 2021 Accepted 19 August 2021

Check for updates

\section{(C) Author(s) (or their} employer(s)) 2021. Re-use permitted under CC BY-NC. No commercial re-use. See rights and permissions. Published by BMJ.

For numbered affiliations see end of article.

Correspondence to Dr Xiaolin Wei; xiaolin.wei@utoronto.ca

\section{ABSTRACT}

Introduction The COVID-19 pandemic has required the rapid development of comprehensive guidelines to direct health service organisation and delivery. However, most guidelines are based on resources found in high-income settings, with fewer examples that can be implemented in resource-constrained settings. This study describes the process of adapting and developing role-specific guidelines for comprehensive COVID-19 infection prevention and control in low-income and middle-income countries (LMICs).

Methods We used a collaborative autoethnographic approach to explore the process of developing COVID-19 guidelines. In this approach, multiple researchers contributed their reflections, conducted joint analysis through dialogue, reflection and with consideration of experiential knowledge and multidisciplinary perspectives to identify and synthesise enablers, challenges and key lessons learnt.

Results We describe the guideline development process in the Philippines and the adaptation process in Sri Lanka. We offer key enablers identified through this work, including flexible leadership that aimed to empower the team to bring their expertise to the process; shared responsibility through equitable ownership; an interdisciplinary team; and collaboration with local experts. We then elaborate on challenges including interpreting other guidelines to the country context; tensions between the ideal compared with the feasible and user-friendly; adapting and updating with evolving information; and coping with pandemic-related challenges. Based on key lessons learnt, we synthesise a novel set of principles for developing guidelines during a public health emergency. The SPRINT principles are grounded in situational awareness, prioritisation and balance, which are responsive to change, created by an interdisciplinary team navigating shared responsibility and transparency. Conclusions Guideline development during a pandemic requires a robust and time sensitive paradigm. We summarise the learning in the 'SPRINT principles' for adapting guidelines in an epidemic context in LMICs. We emphasise that these principles must be grounded in a collaborative or codesign process and add value to existing national responses.

\section{Key questions}

What is already known?

- Infection prevention and control (IPC) guidelines to safely provide high-quality care for COVID-19 have been rapidly created.

- These guidelines are often not role specific and difficult to implement in low-resource settings.

\section{What are the new findings?}

- Codesign between interdisciplinary team members is an important tool towards rapidly developing IPC guidelines for low-resource settings during public health emergencies.

- We propose the following 'SPRINT' principles to guide teams in cocreating IPC guidelines in response to public health emergencies: situational awareness; prioritisation and balance; responsive and reflective; interdisciplinary teams; navigating shared responsibility; and transparency.

What do the new findings imply?

- The ideal guidelines during a public health emergency are those that are contextually relevant, based on best available evidence, and quickly reach health workers to ensure the provision of safe and highquality care.

- Creating such guidelines requires flexible approaches and an interdisciplinary team with both contextual and clinical knowledge.

\section{INTRODUCTION}

The COVID-19 pandemic has required the rapid development of comprehensive guidelines to direct health service organisation and health delivery. Beginning with initial documents based on clinical experience in Wuhan, China, guidance for the prevention, diagnosis and management of COVID-19 have proliferated globally. In particular, infection prevention and control (IPC) guidelines offer clear directions on the arrangement and delivery 
of health services to enable health workers to consistently provide high-quality care. ${ }^{1}$ These guidelines are critical during infectious disease outbreaks to ensure appropriate triage and care of patients, reduce transmission among health workers, and prevent nosocomial spread in healthcare facilities. ${ }^{2}$ In the case of novel infectious diseases, guidance must be rapidly developed based on emerging evidence on disease transmission characteristics including routes of transmission and infectivity. ${ }^{3}$

While there are numerous IPC guidelines from various authorities including the World Health Organization (WHO), there are fewer context and role-specific guidelines with complementary training materials. Many existing guidelines are based on resources found in highincome settings, and from settings in China. There are fewer examples of guidelines that can be adapted and implemented in more resource-constrained settings. ${ }^{4}$ This is particularly concerning given the disproportionate and growing burden of COVID-19 in low-income and middle-income countries (LMICs) and the resource constraints facing their health systems. ${ }^{5}$ Further, these guidelines will continue to be cornerstones of COVID-19 prevention and mitigation for the foreseeable future in these settings due to ongoing global inequities in vaccine delivery. ${ }^{6}$

This study aims to explore the process of rapidly developing IPC guidance and training materials in a pandemic situation. Specifically, we describe the process of adapting and developing role-specific guidelines for comprehensive IPC practices for COVID-19 pandemic management in LMICs.

\section{Research setting}

This work was a part of a larger study, which aimed to adapt, develop, and evaluate role-specific guidelines in the Philippines and modify them for Sri Lanka, with a goal to produce a generic version of the guidelines for further adaptation in other LMICs. Guidelines and associated training videos may be viewed at https://gisldlsphutorontoca/projects-on-covid-19/. Given the rapid timeline, sites were chosen based on existing partnerships. Figure 1 provides the geographical location and description of both countries. Additionally, we believed that understanding the process of development and adaption of COVID-19 guidelines in two different contexts would enable us to synthesise a breadth of lessons on guideline development during a public health emergency. Figure 2 provides an overview of daily reported COVID-19 cases in each country and our timelines during the IPC guideline development process.

\section{The Philippines}

The Philippines recorded its first case of COVID-19 on 7 March 2020 in Cebu province. By 24 April 2020, there were 8212 reported cases, rising to 18997 cases in on 2 June $2020 .^{78}$ As of 24 May 2021, there were over 1.1 million cases and nearly 20000 deaths reported across all 81 provinces. ${ }^{9}$ COVID-19 response measures in the Philippines have focused on border controls, enhanced community quarantine to restrict population mobility, and strategies to bolster the health system such as hiring new health workers and creating isolation facilities. ${ }^{10}$ However, aspects of the initial response were problematic. For example, some of the first available testing kits required overseas processing, which contributed to inequitable access and public hesitancy around COVID-19 testing. ${ }^{11}$ Additionally, while the Department of Health $(\mathrm{DOH})$ released guidelines early in the pandemic in the form of brief updates or 'circulars', these were high level and generalised. ${ }^{12}$ Preliminary interviews as part of our work have indicated that there were gaps in guidance,

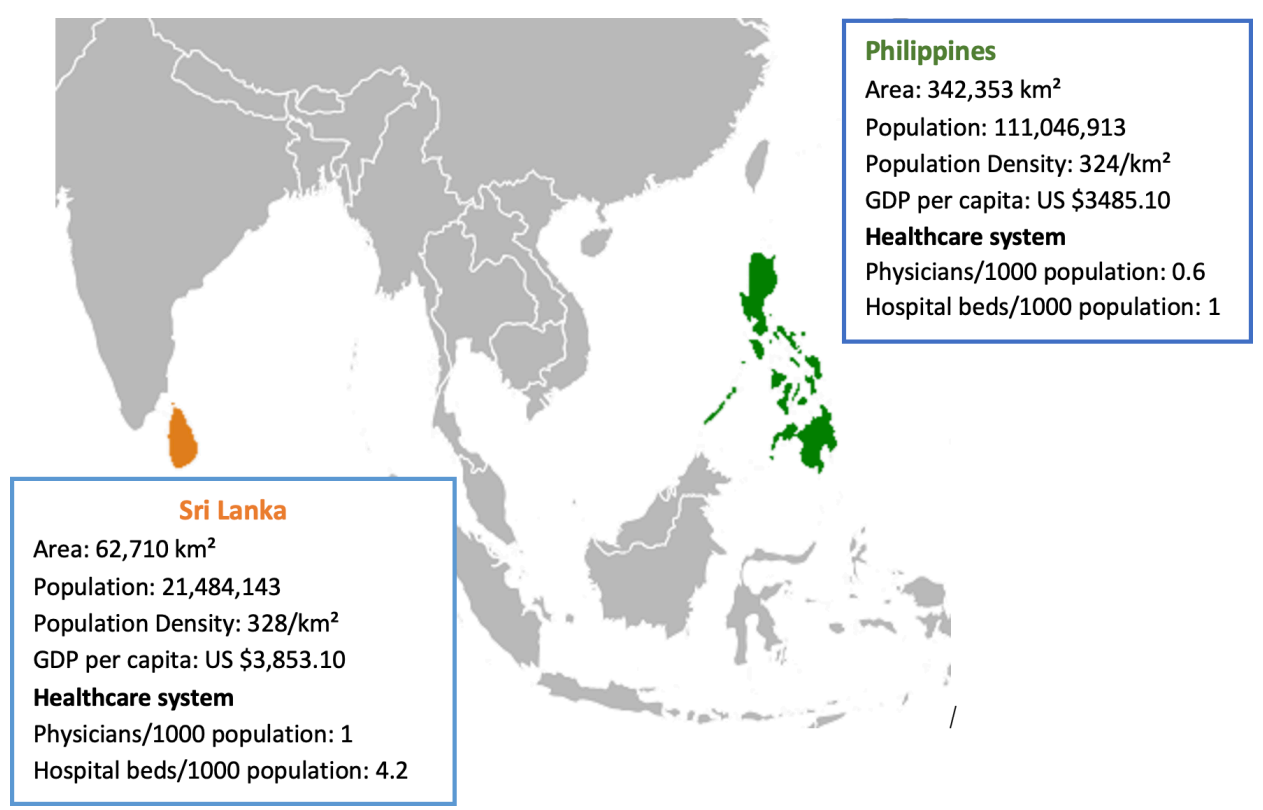

Figure 1 Geography, gross domestic product (GDP), population, and health system context for the Philippines and Sri Lanka. 


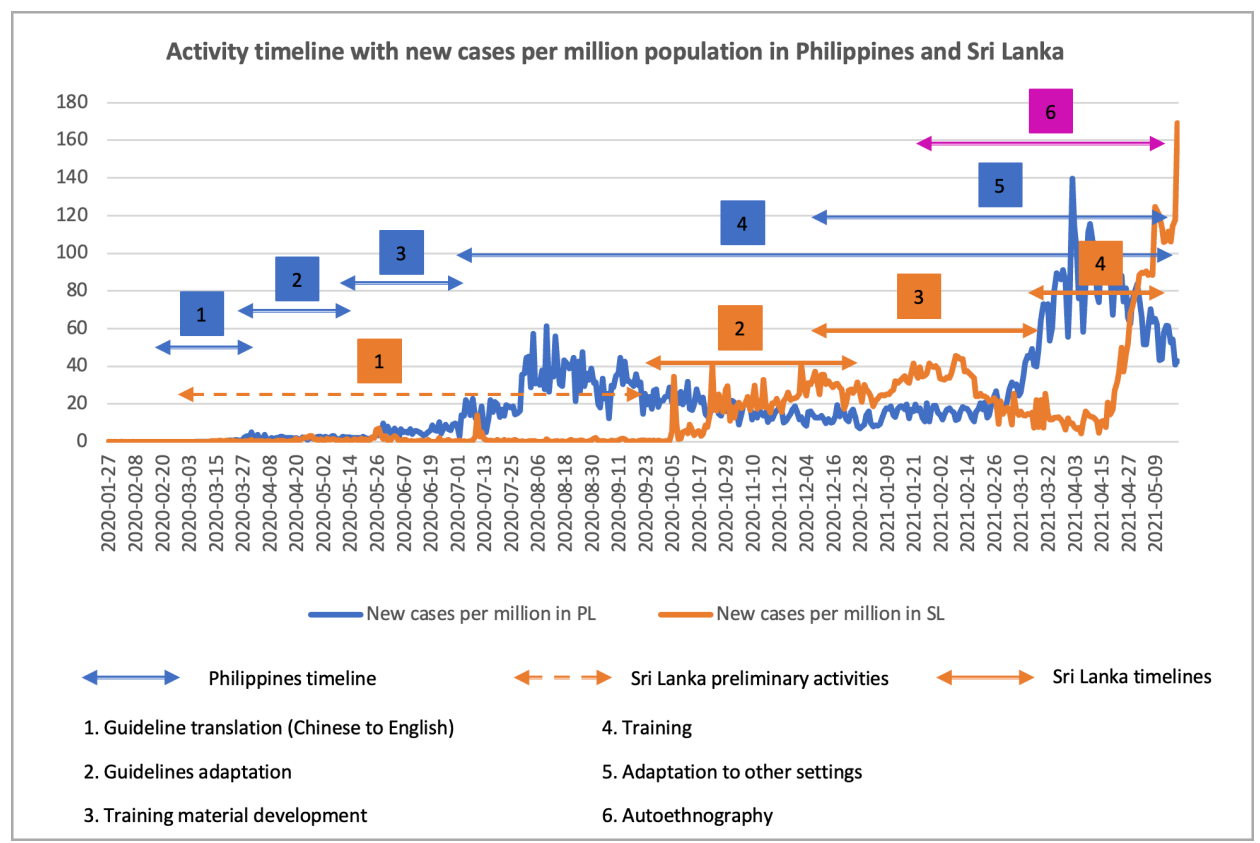

Figure 2 Timeline of activities and daily reported COVID-19 cases per million in the Philippines (PL) and Sri Lanka (SL).

and health workers shared national and other ad hoc guidance through personal channels.

The guidelines development team in the Philippines, including coauthors (LLL and MC), was based at International Care Ministries (ICM). ICM is a Philippinebased non-governmental organisation (NGO) that operates programmes in the Philippines, Uganda and Guatemala. The primary focus of the organisation is the implementation of a community-based poverty reduction programme, which provides education and interventions targeted at health and livelihoods. In the Philippines, ICM has approximately 500 full-time staff, and over 250000 households have participated in ICM programmes. During the strict restrictions on movement enforced during the COVID-19 peaks, ICM health programmes shifted towards addressing acute food security needs and infection prevention education.

In the Philippines, we aimed to develop IPC guidelines for health workers in the following settings: (1) health workers providing care in inpatient healthcare settings, namely public and private hospitals; (2) health workers providing care in outpatient healthcare settings, namely outpatient departments in public and private hospitals; (3) health workers providing care in primary healthcare settings including rural health units and (4) community health workers in community sites, including barangay health stations.

\section{Sri Lanka}

Sri Lanka reported its first case of COVID-19 on 27 January 2020, and the individual was admitted to the National Institute of Infectious Diseases. Guidelines for COVID-19 management were established by the Ministry of Health (MOH) in January 2020. The initial COVID-19 response in Sri Lanka took an aggressive containment approach with strict border controls and the establishment of quarantine centres for returning travellers. All confirmed cases were hospitalised. Sri Lanka leveraged on its pre-existing and robust public health infrastructure and workforce to mobilise comprehensive testing and contact tracing protocols to break chains of transmission in communities. ${ }^{13}$ After initial success containing the first wave, Sri Lanka started reopening the country and the second wave started in October 2020 with decreasing cases loads until April 2021. However, following the Sinhala and Tamil New Year in mid-April, a rapid surge in case incidence and spread of COVID-19 beyond the Western province has increasingly strained resources. By 24 May 2021, there were 164000 reported cases of COVID-19 and 1210 deaths. Although Sri Lanka had regularly updated comprehensive guidance in place at the outset of the project, our existing partners were interested in the feasibility of adapting guidelines and saw value in having training material for role-specific guidelines education for healthcare workers.

The guidelines development team in Sri Lanka included stakeholders from the MOH Sri Lanka with extensive clinical and public health experience (SS, NP) and a researcher in Canada (SR) who completed medical school in Sri Lanka and maintains close connections with Sri Lanka. Partner sites included a district hospital and two MOH areas. In Sri Lanka, we aimed to develop IPC guidelines for health workers in the following settings: (1) health workers providing care in inpatient settings, namely public hospitals; (2) health workers providing primary care; (3) health workers providing care in outpatient settings, namely in outpatient departments of public hospitals and (4) field health workers providing community care. 


\section{METHODS}

This is a collaborative autoethnographic study where authors used their own experiences to expand our understanding of adapting and developing role-specific guidelines for comprehensive care of an emerging infectious disease in LMICs. Autoethnography uses a researcher's experiences as primary data to analyse and interpret sociocultural meanings of events. ${ }^{14}$ Collaborative autoethnographic research is a method whereby multiple researchers contribute their reflections on an event or experience, conduct joint analysis of the material through dialogue, reflection and with consideration of experiential knowledge and multidisciplinary perspectives. ${ }^{15}$ Team members actively involved in the adaptation and development of these guidelines in Canada, the Philippines, and Sri Lanka were invited to participate in a 1-hour interview to explore their perceptions and experiences with developing the guidelines, or provide a free form written response to the prompt 'please describe the guideline development process from your perspective in your own words-including any specific examples of challenges or strengths of the development process.'

Participants were recruited in January 2021 after completion of IPC guideline development in both countries following several rounds of iteration. Nine team members participated in interviews, and two provided lengthy written replies. We provide a schematic of the team and their roles in online supplemental file 1. Team members were invited to participate as coauthors and were provided written details on the study including details on confidentiality of responses. Interviews were held via videoconference and team members were explained the study at the start of the interview and provided additional verbal consent. Interviews were conducted by a team member in Canada $(\mathrm{VH})$, a $\mathrm{PhD}$ candidate who is experienced in qualitative methods and acted as study coordinator between both teams since February 2020, thus establishing deep rapport with team members. Accounts were transcribed in full and anonymised. Based on these transcripts VH curated an 'overarching narrative' of the development process. The lens that $\mathrm{VH}$ brought to data collection and curation, meaning the condensation, categorisation, and creation of a cohesive master narrative from the interviews, was reviewed through a critical lens both by VH and SR in a process of active discussion during the curation process. This critical discussion included reflexive discussion around positionality on the team and in writing this manuscript (online supplemental file 2). Part of this reflection was considering the foreign gaze and to what extent a collaborative autoethnographic approach and our presentation of the autoethnography perpetuates or addresses what can be called 'epistemic injustices' in global health research and writing. ${ }^{16} 17$ This curated narrative was reviewed by all team members who provided candid and nuanced feedback during several videoconference meetings, numerous text messages, and written feedback on drafts to ensure coherence in light of multiple voices, perspectives and lived experiences.
Once the document reflected a shared perception of the overall process, three team members, $(\mathrm{VH}, \mathrm{SR}$ and $\mathrm{NP})$ developed themes on barriers and enablers based on transcripts, written data and the overarching narrative. ${ }^{18}$ This process involved multiple discussions and reflection on the text by the three researchers to identify themes that captured barriers and enablers. The resultant themes were then disseminated to all authors who confirmed and expanded on them through multiple emails and shared drafts. This process involved negotiation between the team on how to articulate the narrative and principles, without silencing any voices or minimising any experiences. We then synthesised our collective lessons learnt into key principles. This process also involved negotiation and reflection among the research team through multiple meetings to identify and refine the principles.

Patients or the public were not involved in the design, or conduct, or reporting, or dissemination plans of our research.

\section{RESULTS}

We organise our results by first offering a narrative of the steps in developing our context-specific and rolespecific guidelines and training materials beginning with 'the process', that is (1) developing a baseline set of guidelines; (2) adapting these to the Philippines context and (3) again adapting to the Sri Lanka context. We then reflect on our experiences and offer barriers and enablers to guidelines development.

\section{Sources and adaptation of guidelines}

In early 2020, there were few clinical guidelines for the diagnosis, treatment and management of patients with COVID-19. As a foundation for our initial draft guidelines, in February 2020, we drew on guidelines from the WHO and China's national COVID-19 response. We translated the guidelines from China and aimed to adapt them to the health system context of the Philippines and then, based on learnings from this adaption process, again adapt them to the health system context of Sri Lanka. To do so, we partnered with colleagues from both countries to develop role-specific guidelines and complementary training materials for health workers in each country. Our multinational teams organised into a Philippines Working Group and Sri Lanka Working Group jointly connected by a mutual team based in Canada with clinical expertise from the UK to support with guidance design, formatting and reviewing. A shared coordinator connected both working groups .

The team created preliminary drafts of the guidelines and their associated 'desk guides.' Desk guides are a condensed and easily accessible clinical tool to provide guidance to front-line healthcare workers based on experience from other disease control (http:// comdis-hsd.leeds.ac). To better understand emerging guidelines issued by WHO and national bodies globally, 
we conducted a grey literature review. ${ }^{19}$ Some team members wondered if we should have a thorough search of the academic literature to build our awareness and understanding of the breadth of emerging guidelines, particularly as the virus spread globally and China ceased to be the sole epicentre and knowledge hub. One team member mentioned that 'whatever recommendation we give, the evidence should be clear.' However, there was tension between wanting definitive evidence and the often-changing guidance as scientists learnt more about the virus and disease characteristics. Given the proliferation of SARS-CoV-2 studies, we performed a comprehensive literature search with a librarian, which yielded 3226 references, many of them preprints. Finding and disseminating the literature so that team members could update the guidelines with emerging evidence from research and practice quickly became an overwhelming task, as one team member commented 'it was like trying to drink water from a fire hydrant.' To cope with this, we began to structure literature check-ins, at first every 2 weeks, however, once there was fewer emergent COVID-specific IPC evidence, we reviewed the literature every 2 months. Additionally, research team members set up keywordspecific alerts and followed WHO briefings. Clinical team members kept abreast of any updates at their institutions or through their professional associations that may be useful for the study.

\section{Developing guidelines for the Philippines}

Here, we outline the process of developing guidelines for health workers in four unique health delivery settings in the Philippines: (1) Management Guidelines for COVID-19 Infection Prevention and Control in the Healthcare Setting; (2) Outpatient Consultation Desk Guide; (3) Primary Care Health Worker Desk Guide and (4) Barangay (Community) Health Worker Desk Guide.

Given the rising case numbers at the start of the project in March 2020, there was a sense of urgency in creating the guidelines. Furthermore, in the Philippines, the health system is fragmented with structural challenges to information dissemination among health facilities, namely underdeveloped information technology systems to ensure consistent information reaches all health facilities, as well as information asymmetries between private and public providers, as well as geographical disparities in available resources. ${ }^{20}$ Importantly, our partner NGO had an immediate need for guidelines and training to protect their front-line staff in communities. These factors necessitate a rapid and flexible approach to create and deliver usable and context-specific guidelines. To adapt available guidelines to the Philippines context, the team in the Philippines with support from the team in Toronto reviewed the preliminary guidelines draft, cross checked with the translated guidelines from China, the WHO guidelines, and locally available Philippines DOH guidelines. An important part of this process was then further adapting the guidelines based on the needs of additional groups of health workers identified by our partner organisation.
For example, we adapted the guidelines and training materials for tuberculosis treatment partners (kagabays) who provide care in the community.

These documents were discussed at length over emails and through videoconference meetings with clinical consultation from staff affiliated with our partner organisation in the Philippines and the Canada team. For example, some members of the team worked in teaching hospitals in Toronto and were aware of issues with and adherence to the initial Canadian guidelines, thus they posed questions about how the guidelines could feasibly be implemented in low-resource settings. Teams in both locations reviewed the emerging scientific literature, highlighted questions, and met regularly over videoconference to resolve these uncertainties. Meetings focused on discussion considering both the evolving evidence on the nature and management of COVID-19, as well as the contextual differences between China and the Philippines. These meetings required the team to strike a balance between the objectives of the project, the COVID19-related IPC materials available, and the situation in the Philippines. This was achieved through an explicit awareness of our evolving understanding of the project, and negotiation of how to proceed despite uncertainty and challenges. This process challenged hierarchies and required a negotiation of power away from traditional research seniorities and roles and towards being reflexive to the needs of those in the field and indeed what was even possible in the research setting. Once the guidelines content was reviewed, another member of the team took the lead to make the guidelines concise and user friendly. The member responsible for copy editing and formatting also strived to check the accuracy of the latest version to ensure it was updated with key guidance from the WHO, US Centers for Disease Control and Prevention (CDC) or national guidelines. We also implemented a final check-up process based on a review against most recent national guidelines and final read through by a healthcare worker affiliated with ICM to see if the guidelines fit into the resources available and requirements in the Philippines.

Concurrently, we developed training materials. However, due to 'lock-down' restrictions on movement, we were unable to provide in-person training at many sites, and thus created materials to support virtual sessions. As such, ICM staff engaged with a film crew and celebrity to direct, film, and produce a series of training videos. A popular singer, who is also a registered nurse, played a major role in introducing and promoting the training videos which garnered credibility and held the attention of viewers in the Philippines. The production of these videos occurred during strict public health measures in the area. These circumstances required the Philippines team to be creative and flexible to complete filming, while still abiding by all public health measures, and ensuring the safety of themselves and others. The outcome of these efforts was a comprehensive suite of COVID-19 guidelines training materials and videos. ${ }^{2}$ 
These videos offered training and in-depth information on each chapter of the guidelines. Each video chapter included informational slides describing the guidelines, expert narration on the guidelines, and role play scenarios to illustrate the guidelines.

\section{Adapting the guidelines for Sri Lanka}

Here, we outline the process of developing guidelines for health workers in four unique health settings in Sri Lanka: (1) Management guidelines for COVID-19 infection prevention and control in the healthcare setting; (2) Primary care health worker desk guide in the context of COVID-19; (3) Desk guide for Outpatient Consultation in Hospital in the Context of COVID-19 and (4) Desk guide for Field Health Workers in the Context of COVID19.

The desk guides prepared by the Philippines team were shared with the Sri Lanka team in March 2020. During that time, the situation in Sri Lanka was fairly controlled with few cases and deaths. The 'Provisional Clinical Practice Guidelines on COVID-19 suspected and confirmed patients' and a 'Manual for hospital preparedness for COVID-19' were released and updated by the MOH in Sri Lanka. Our work intended to complement and enhance these materials.

In mid-2020, we submitted for ethics approval from the $\mathrm{MOH}$ and strengthened relationships with local partners. We anticipated bureaucratic delays when the pandemic situation worsened while seeking approvals. To better understand what elements would be most useful we conducted a needs assessment among health system stakeholders (results forthcoming). From this needs assessment our site partners highlighted that we needed to include guidelines and materials specifically for ancillary staff, such as sanitation workers in hospital settings. The initial draft adapting the Philippines guidelines to the Sri Lanka context, which had been drafted in April 2020, was of limited use given the evolving evidence and rapidly changing situation in Sri Lanka. Thus, we begin to again update the guidelines. This process involved six rounds of updating between team members in Canada and Sri Lanka to ensure accuracy, and then further review and formatting. By this time, in Sri Lanka, the second wave of infections had begun and there was increasing urgency and need for training material. This final review and editing process was an intense process that involved multiple telephone calls and videoconferences and took two weeks to complete.

By the end of November 2020, we had edited the guidelines to reflect new information and aligned with the latest high-level guidelines from Sri Lanka. At this time, the epidemic in Sri Lanka became overwhelming, and the review process became very hard for the team in Sri Lanka. Fortunately, we had good communication links with the partner sites and were able to pilot the guideline and training sessions. But it was tough, as a team member stated, 'Honestly, if we did not know them personally, they would have had no reason to work on this at $9 \mathrm{pm}$ after they finish their 14-hour work-day.'

Finally, stakeholder interviews were conducted in December 2020 and January 2021 to adapt the guides and identify specific needs for the training videos. We realised even at the late stage of preparing the videos that some of the technical information changed with emerging information and had to put in a disclaimer stating that in any pandemic situation IPC guidelines continue to be modified with newly emerging evidence; however, basic IPC principles remain the same.

\section{Team reflections: enablers and challenges to guidelines development}

Based on our experiences, we summarise key enablers and challenges to the development of context-informed and role-specific guidelines for the treatment and management of COVID-19. We present these as themes created from our autoethnographic process (table 1).

A key enabler described was flexible leadership that aimed to empower the team to bring their expertise to the process. While this approach sometimes caused team members to feel like roles and responsibilities were not well-defined, it also challenged team members to seek broad sources of evidence and offer creative solutions to improve the guidelines. For example, one team member leveraged on her clinical and design skills to lead on making the guidelines user-friendly. This enabler closely related to the sense of shared responsibility and equitable ownership the process engendered. Researchers used their professionalism and expertise to take ownership of the guidelines each time it came to them for review and improvement. Finally, the interdisciplinary team brought multiple perspectives and experiences to the guidelines creation process. This included both professional experiences, as well as lived experience given that many team members were trained, previously practised or actively live and work in LMICs. In addition, the team worked closely and sought clinical guidance from frontline staff in both settings. Given that front-line staff were responding to the pandemic and had limited capacity to work on guideline development, this was a delicate task. As such, the guidelines development team relied on preexisting relationships, sometimes informal ones, to find out specifics. Getting timely feedback was at times challenging, particularly when trying to make new connections, which sometimes felt like overstepping or imposing on busy front-line staff.

The development process, clearly, was not without challenges. A major challenge was interpreting and incorporating the local context. Available guidance at the time was either high level and generic or was too resourceintensive for the context. For example, the guidelines from China had CT scans as a diagnostic tool, an unfeasible assessment method in our contexts. Within the team, there was also a heightened awareness that some team members who had experience creating guidelines or other clinical expertise, did not have contextual 
Table 1 Enablers and challenges to COVID-19 guidelines development

Enablers

$\begin{array}{ll}\text { Leadership } & \text { 'I cannot achieve this without input from different team members. I think my role was much overarching the } \\ \text { guided by } & \text { project, for example, I think one of the roles is that I need to identify the different areas we have to work, and } \\ \text { flexibility and } & \text { then to add the people who can work in this area.' } \\ \text { empowerment } & \begin{array}{l}\text { 'Looking back, it was a hard time for anyone to be writing guidelines...I reached out to [clinical colleague] } \\ \text { who has a lot of clinical experience, she's really busy looking after the running all of our health programs... } \\ \text { but she had insight into system here and her own resources and her own networks which were helpful.' }\end{array}\end{array}$

Shared 'I think there is an amalgamation of work of different projects, so the documents weren't fully developed, responsibility and are in the draft form so going back and forth with team members to finalise either the wording or doing through equitable ownership copy edits of the guidelines themselves...That was the process going back and forth.' 'When each team member had the guidelines, it was like it was their responsibility, to check and double check everything and work together to confirm the contents. There was hesitation by some non-clinical team members but ultimately, by each team member from their unique disciplinary perspective and experience taking responsibility and flagging issues or asking questions, it created this shared ownership.'

'I think one of the tricky things about the nature of guidelines is you're giving advice to doctors and nurses and it's also guidelines in the middle of an international crisis when the evidence is unclear, so I think what was hard was as I pass this 'hot potato' around, who has the authority to have the final say on this? If I imply something wrong, then what happens?'

$\begin{array}{ll}\begin{array}{ll}\text { Interdisciplinary } \\ \text { team and }\end{array} & \text { 'We had connections with the Ministry of Health, pandemic management team, all public health networks } \\ \text { collaboration } & \text { knowing the system helped us to translate and modify it.' } \\ \text { with local } & \text { 'I feel you have to speak to the ground level because the upper national level guideline is different. The } \\ \text { experts } & \begin{array}{l}\text { activity at ground level is different from country to country so you have to take the inputs from the grassroot } \\ \text { level then adapt it to your structure... It'll be easier or productive, or else, the documents we make will not be } \\ \text { practiced in the field.' } \\ \text { 'And I think for a few on the team they really wanted to help and had a lot of skills to offer but there was that } \\ \text { removed feeling if you were non-clinical, there was a feeling of being an 'outsider' to the medical field' }\end{array}\end{array}$

Challenges

Interpreting and 'I was looking at the WHO interim guidelines...I was looking at some CDC guidelines as well...It's a bit incorporating different when you apply it to a low-income country context, there's not as many supplies, there's more context challenges that you have to think about...A lot of the guidelines were coming from high-income countries like the UK or USA. Even though we had local input, I wonder if we are missing guidelines from elsewhere because they are harder [for us] to access.'

'The China guideline said that even if they are negative on PCR, they may still have the disease so do a CT scan, which was part of the guidelines for them. That was questioned by Sri Lanka, why we have to do that?'

'The most challenging thing was that I knew little of the local context and local practice of relevant staff. What I could do was to organise the [existing] contents based on my previous experience in China and developed a first draft for further revision by local partners. During the process, I also added some sections suggested by the local partners during regular meetings, (mental health support and corpse disposal) and updated sections according to latest national guidelines... We can't just cut and paste under different situations. So you need to speak to the ground level people, then according to that, amend the guideline.'

Tensions between the ideal guidelines versus timeliness and usability
'I felt that I was going at a snail's pace with the transcript because I kept finding things in the documents and the documents were pretty large...I wanted to defer to the health experts on the content. Knowing that it needs to get into a published form... was probably the biggest challenge I faced at that time.'

'A large part was taking a huge amount of information... and diluting it down, making it readable and digestible and also creating desk guides from that as well to make it more digestible. It was big but, a lot of it was taking something from larger and abstract and make it more relevant and narrowing it down and digestible for people to use on a daily basis.'

'When you write guidelines, you should be open and honest at that moment, and clearly state that when things change, we will update the guidelines.'

'We went into the process thinking this would be a very positivist exercise, but it's much more constructivist. Things are evolving and emerging - the evidence, the situation on the ground, the virus itself. Developing comprehensive guidelines in this situation requires a bit of a mindset shift towards the 'good enough' model where you get something out that's good enough given all known information and best clinical practices and are clear with each other and with your end users that it will be updated pending new evidence.' 
Table 1 Continued

$\begin{array}{ll}\begin{array}{l}\text { Updating } \\ \text { and adapting } \\ \text { with evolving } \\ \text { information }\end{array} & \begin{array}{l}\text { 'The frequently updated local national guidelines made the guideline development process more } \\ \text { 'We had already printed [the guidelines] and then there are changes...I guess that the fluidity [to update the } \\ \text { guidelines] was kind of lost. I like a real-time sense of where we were in training vs updating the guideline. } \\ \text { And which is also understandable with things coming out at different times so, I don't see that as a fault. But } \\ \text { content evolving, I think that was [a challenge]. Just getting it in before the next training, needing to know } \\ \text { [any changes].' }\end{array} \\ \begin{array}{l}\text { The impact of } \\ \text { the pandemic }\end{array} & \begin{array}{l}\text { 'In a pandemic, our ability to push forward the work is really hindered by the emergencies. There have been } \\ \text { a number of emergencies in Philippines and Sri Lanka which has delayed our project. So I don't think that }\end{array} \\ & \begin{array}{l}\text { 'It felt like there were limited staff and a lot of volatility during the initial phase... there were all of these } \\ \text { intense situations happening that were underlying the process...so it was good that we had a bigger team, } \\ \text { the intensity of the situation made us work together well and support each other better and have a lot of } \\ \text { conversations' }\end{array}\end{array}$

expertise, and that some team members with contextual expertise, did not feel confident or that it was appropriate for them to provide clinical insights. These challenges required the team to have multiple discussions and ongoing meetings to triangulate evidence, experience, and clinical expertise. Given that the pandemic was rapidly unfolding this led to tension between the ideal guidelines vs timeliness and usability. This was particularly the case in the Philippines where health worker trainings were conducted nearly immediately after the guidelines were printed. A 'good enough' approach based on best available evidence and practices was taken overall, together with transparency that there would be updates and iterations as new evidence became available. This approach emphasises the importance of getting the right information out to front-line workers and staff who need it most, as opposed to waiting to create the 'ideal' guidelines, an abstract goal at odds with the immediate needs of health workers. Updating and adapting the guidelines with evolving information was a challenge given the vast amounts of new knowledge generated in early 2020 on the management of COVID-19. Further, the guidelines process was challenged by the impact of the pandemic, which necessitated rapid development and implementation in situations that were at times rapidly deteriorating. Additionally, the researchers themselves faced pandemic-related challenges including health, mental well-being, and increased caregiving and professional responsibilities. These factors, while challenging, were also opportunities for greater team cohesion and personal connection around shared struggles. This ultimately strengthened our approach and solutions to challenges faced in creating the guidelines during the early stages of the pandemic.

\section{DISCUSSION}

The global emergence of COVID-19 in early 2020 demanded rapid, easily usable, and context-specific clinical guidelines to ensure the safety of health workers and patients. In this autoethnographic study, we have elaborated on our process of developing context-specific and role-specific guidelines and reflected as a team on barriers and enablers to illustrate key lessons learnt during this process. We now offer a synthesis of our process and lessons learnt in the form of the 'SPRINT' principles, a novel framework for teams aiming to create guidelines in response to public health emergencies caused by emerging infectious diseases (figure 3). SPRINT stands for 1) Situational awareness; 2) Prioritisation and balance; 3) Responsive and reflective; 4) Interdisciplinary; 5) Navigating shared responsibilities; and 6) Transparency. While these lessons were synthesised from experiences in LMICs, the principles are applicable and important in all resource-constrained settings responding to an epidemic.

First, we emphasise the importance of situational awareness. This includes attention to the epidemiological

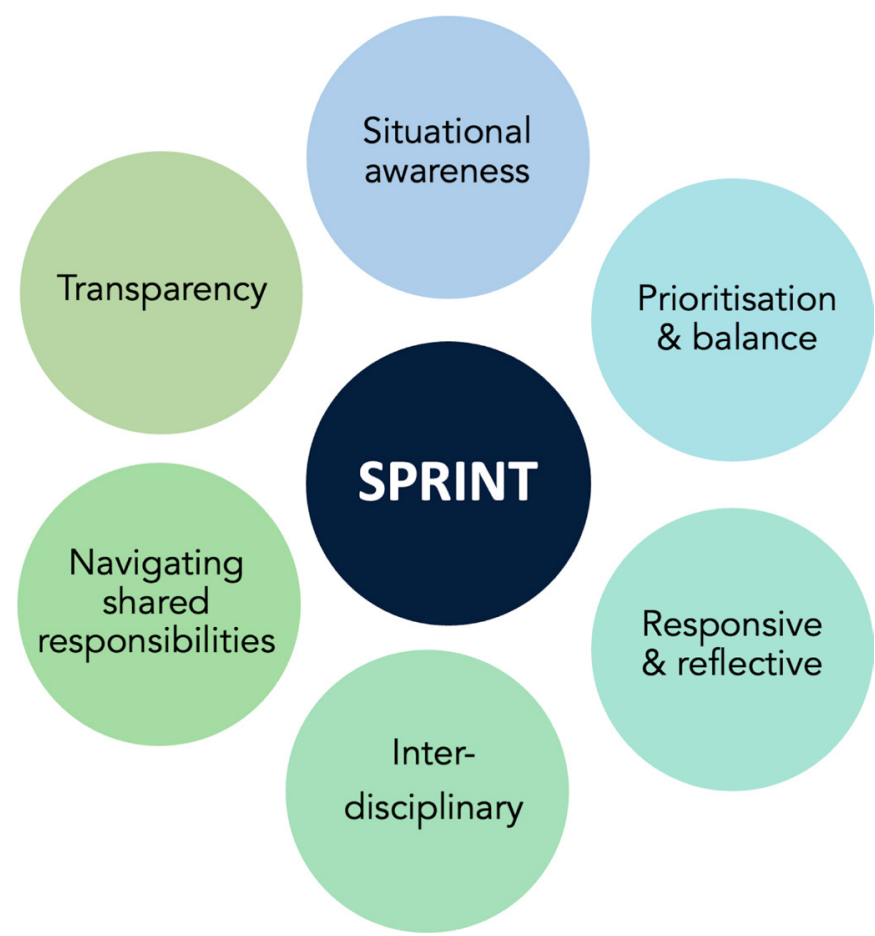

Figure 3 Lessons learnt from the development of COVID-19 guidelines. 
situation, current guidelines being used, as well as the contexts where guidelines will be applied, with special attention to non-clinical settings that may require guidance. In the Philippines, this process resulted in the identification of several settings where guidelines for community health services were needed but didn't exist. We identified that NGO health workers were underserved but would require guidelines and training to protect themselves and mitigate the spread of COVID-19 in communities. In Sri Lanka, we received inputs while seeking ethical approval for the project, that it must include guidelines for ancillary staff such as cleaners in health facilities. As such, teams should work closely with front line and other health system stakeholders. Early on the team should prioritise and balance competing demands to set goals for the area of care the guidelines will address at an early stage. This should inform research such as literature searching, and the development priorities.

Throughout the process, teams should be prepared to be responsive and reflective to an evolving situation with continuous inputs of new evidence and best practices that at times may contradict previous evidence. Teams should also be prepared to negotiate new power dynamics or reimagine existing clinical or research hierarchies to ensure that the team is able to be responsive to a changing landscape and not internally uphold structures that hinder reflection and development. Particularly during the development phase, it requires an interdisciplinary team to take a 'good enough' approach in line with best available evidence and prevailing clinical practice. Importantly, teams should comprise local staff who know the setting, clinical and epidemiological experts, and a literature reviewer who passes information to the team. Other important skillsets include the behavioural and social sciences, design skills, and project management. There is a need for ownership and teams should foster a culture of navigating shared responsibility, where each team member takes ownership of the guidelines when it is their turn to review. This should be supported by flexible leadership and a focal point person tasked with disseminating emerging evidence to the team. Finally, teams should make efforts to transparently document the evidence informing guideline creation with full acknowledgement that the information will likely be updated as new information or best practices emerge.

During public health emergencies, particularly those caused by a novel infectious disease, clinical guidelines must be developed rapidly while minimising any loss of rigour and utility. This contrasts with developing clinical guidelines under routine circumstances, where time is afforded to draw on and systematically review an ample evidence base. ${ }^{21}$ Indeed, during a public health emergency, guidelines are often initially selected based on a familiar model of care, drawing on plans for influenza, or for Severe Acute Respiratory Syndrome or Middle Eastern respiratory syndrome events. While other countries, particularly LMICs, based their approaches and recommendations on past experiences with cholera or Ebola. However, as information emerges on routes of transmission and disease characteristics, new diseasespecific guidance must be developed, typically the remit of the WHO or National CDC. However, these guidelines are then synthesised and adapted, making them context and role specific as per the country's health system.

Using a collaborative autoethnographic method, our study provides an important description and critical reflection on the guideline development process by a multinational and multidisciplinary team. An important limitation of this work is that we were unable to include the needs and experiences of community members in developing IPC guidelines. Teams embarking on this work should actively pursue avenues for community involvement in design processes. Further expansion on this work could use the SPRINT principles as complementary to codesign frameworks and approaches for patient involvement such as experience-based codesign or others, in the context of IPC guideline creation for emerging infectious hazards. ${ }^{22}$ Another limitation to deep and inclusive codevelopment grounded in the research setting is that many researchers in our team were based at institutions in high income countries. This could potentially skew the overarching narrative we present to uphold dominant (eg, Western or high-income country) discourses. While the SPRINT principles are key considerations for teams undertaking similar work, we also underscore that these principles must be grounded in a codevelopment approach. Our team understood codevelopment to be an iterative process of shared ownership that sought to create guidelines with partners, rather than for partners. The strength of this approach became apparent when our team was able to adapt the guidelines based on feedback. This included creating guidelines for at-risk roles identified by partners as underserved by current guidelines in each context, such as front-line NGO community workers in the Philippines or ancillary hospital staff in Sri Lanka.

\section{CONCLUSIONS}

Guideline development during a pandemic requires a robust and time sensitive paradigm. The SPRINT principles are particularly useful for adapting guidelines to LMICs and other settings using an approach grounded in situational awareness, prioritisation and balance, which are responsive to change, created by an interdisciplinary team navigating shared responsibility and transparency. We emphasise that these principles must be grounded in codesign and add value to existing national responses. In this study, we outline a process and principles, which provide a foundation for ongoing collaboration during the COVID-19 pandemic. Sharing this process and these principles is important given emerging evidence on the management of COVID-19, the threat of variants of concern, and ongoing vaccination efforts, which also rely on IPC guidance. 
Looking ahead to future emerging infectious threats, we must collectively act to prevent outbreaks from becoming pandemics. ${ }^{23}$ The SPRINT principles provide a blueprint for rapid creation of guidelines to safeguard health workers and communities as part of pandemic and emergency preparedness and response.

\section{Author affiliations}

${ }^{1}$ Institute of Health Policy, Management and Evaluation, University of Toronto, Toronto, Ontario, Canada

${ }^{2}$ Dalla Lana School of Public Health, University of Toronto, Toronto, Ontario, Canada

${ }^{3}$ Department of Emergency Medicine, SickKids, Toronto, Ontario, Canada

${ }^{4}$ Temerty Faculty of Medicine, University of Toronto, Toronto, Ontario, Canada

${ }^{5}$ International Care Ministries Inc, Manila, Philippines

${ }^{6}$ Ministry of Health, Colombo, Sri Lanka

${ }^{7}$ School of Public Health Sciences, University of Waterloo, Waterloo, Ontario, Canada ${ }^{8}$ Nuffield Centre for International Health and Development, University of Leeds, Leeds, UK

Contributors VH and SR designed and conceptualised the study. VH collected the data provided by coauthors. VH, SR and NP analysed the data. VH, SR, NP, SG, ZZ, MC, LLL, SS, JW, and XW reviewed the analysis. VH and SR wrote the first draft of the manuscript with input from NP, SG, ZZ, MC, LLL, SS, JW and XW. All authors contributed to the final draft.

Funding This work was funded by the Canadian Institute of Health Research (ClHR) and International Research and Development Agency (IDRC) (439835).

Map disclaimer The inclusion of any map (including the depiction of any boundaries therein), or of any geographic or locational reference, does not imply the expression of any opinion whatsoever on the part of BMJ concerning the legal status of any country, territory, jurisdiction or area or of its authorities. Any such expression remains solely that of the relevant source and is not endorsed by BMJ. Maps are provided without any warranty of any kind, either express or implied.

Competing interests None declared.

Patient consent for publication Not required.

Ethics approval Ethical approval for this study was obtained from the Office of Research Ethics at the University of Toronto (Ref: 20291); the Independent Ethics Committee at De La Salle Medical and Health Sciences Institute (Ref: 2020-14-06A); and the Sri Lanka Medical Council (ERC 20-013).

Provenance and peer review Not commissioned; externally peer reviewed.

Data availability statement All data relevant to the study are included in the article or uploaded as online supplemental information.

Supplemental material This content has been supplied by the author(s). It has not been vetted by BMJ Publishing Group Limited (BMJ) and may not have been peer-reviewed. Any opinions or recommendations discussed are solely those of the author(s) and are not endorsed by BMJ. BMJ disclaims all liability and responsibility arising from any reliance placed on the content. Where the content includes any translated material, BMJ does not warrant the accuracy and reliability of the translations (including but not limited to local regulations, clinical guidelines, terminology, drug names and drug dosages), and is not responsible for any error and/or omissions arising from translation and adaptation or otherwise.

Open access This is an open access article distributed in accordance with the Creative Commons Attribution Non Commercial (CC BY-NC 4.0) license, which permits others to distribute, remix, adapt, build upon this work non-commercially, and license their derivative works on different terms, provided the original work is properly cited, appropriate credit is given, any changes made indicated, and the use is non-commercial. See: http://creativecommons.org/licenses/by-nc/4.0/.
ORCID iD

Lincoln L Lau http://orcid.org/0000-0002-7386-4104

\section{REFERENCES}

1 World Health Organization. WHO Handbook for Guideline development. World Health Organization, 2010.

2 Global Implementation Science Lab. COVID-19 Deskguides for healthcare workers 2021. Available: https://gisl.dlsph.utoronto.ca/ projects-on-covid-19/

3 Norris SL, Sawin VI, Ferri M, Reques Sastre L, et al. An evaluation of emergency guidelines issued by the World Health Organization in response to four infectious disease outbreaks. PLoS One 2018;13:e0198125.

4 Maaløe N, Ørtved AMR, Sørensen JB, et al. The injustice of unfit clinical practice guidelines in low-resource realities. Lancet Glob Health 2021;9:e875-9.

5 Hopman J, Allegranzi B, Mehtar S. Managing COVID-19 in low- and middle-income countries. JAMA 2020;323:1549-50.

6 Choi EM. COVID-19 vaccines for low- and middle-income countries. Trans R Soc Trop Med Hyg 2021;115:447-56.

7 World Health Organization. Coronavirus disease (COVID-19) situation report 25 Philippines 29 April 2020. 202029 April 2020.

8 World Health Organization. Coronavirus disease (COVID-19) situation report 37 Philippines 2 June 20202020.

9 Our World in Data. Coronavirus (COVID-19) cases 2021. Available: https://ourworldindata.org/covid-cases

10 Vallejo BM, Ong RAC. Policy responses and government science advice for the COVID 19 pandemic in the Philippines: January to April 2020. Prog Disaster Sci 2020;7:100115.

11 Buhat CAH, Duero JCC, Felix EFO, et al. Optimal allocation of COVID-19 test kits among accredited testing centers in the Philippines. J Healthc Inform Res 2021;5:54-69.

12 Republic of Philippines Department of Health. Interim guidelines on the management of person under monitoring (PUMs) suspected with COVID 2019 for home quarantine. Contract No.: no. 2020-0105 2020.

13 Ratnapalan S, Perera N, Wei X. Current experience and future challenges of COVID-19 in Sri Lanka: an autoethnographic study. Public Health Open Access 2020;5.

14 Chang $\mathrm{H}$. Autoethnography in health research: growing pains? Qual Health Res 2016;26:443-51.

15 Ellis C, Adams T, Bochner A. Autoethnography: an overview. Forum Qualit Res 2011;12:10.

16 Bhakuni H, Abimbola S. Epistemic injustice in academic global health. Lancet Glob Health 2021 doi:10.1016/S2214109X(21)00301-6

17 Abimbola S. The foreign gaze: authorship in academic global health. BMJ Glob Health 2019;4:e002068.

18 Braun V, Clarke V. Using thematic analysis in psychology. Qual Res Psychol 2006;3:77-101.

19 Haldane V, Zhang Z, Abbas RF, et al. National primary care responses to COVID-19: a rapid review of the literature. BMJ Open 2020;10:e041622.

20 Dodd W, Kipp A, Nicholson B, et al. Governance of community health worker programs in a decentralized health system: a qualitative study in the Philippines. BMC Health Serv Res $2021 ; 21: 451$.

21 Umscheid CA, Agarwal RK, Brennan PJ, et al. Updating the guideline development methodology of the healthcare infection control practices Advisory Committee (HICPAC). Am J Infect Control 2010;38:264-73.

22 Green T, Bonner A, Teleni L, et al. Use and reporting of experiencebased codesign studies in the healthcare setting: a systematic review. BMJ Qual Saf 2020;29:64-76.

23 Sirleaf EJ, Clark H. Report of the independent panel for pandemic preparedness and response: making COVID-19 the last pandemic. Lancet 2021;398:101-3. 